\title{
ON MAGNUS INTEGRATORS FOR TIME-DEPENDENT SCHRÖDINGER EQUATIONS
}

\author{
MARLIS HOCHBRUCK* AND CHRISTIAN LUBICH**
}

\begin{abstract}
Numerical methods based on the Magnus expansion are an efficient class of integrators for Schrödinger equations with time-dependent Hamiltonian. Though their derivation assumes an unreasonably small time step size as would be required for a standard explicit integrator, the methods perform well even for much larger step sizes. This favorable behavior is explained, and optimal-order error bounds are derived which require no or only mild restrictions of the step size. In contrast to standard integrators, the error does not depend on higher time derivatives of the solution, which is in general highly oscillatory.
\end{abstract}

1. Introduction. We study numerical integrators for Schrödinger equations with time-dependent Hamiltonian,

$$
i \frac{d \psi}{d t}=H(t) \psi, \quad \psi\left(t_{0}\right)=\psi_{0}
$$

The computational Hamiltonian $H(t)$, which is a finite-dimensional hermitian operator, is typically the sum of a discretized negative Laplacian and a time-dependent potential. As the discretization of an unbounded operator, $H(t)$ can be of arbitrarily large norm.

Magnus integrators are an interesting class of numerical methods for such problems $[3,12]$. Though the error behavior of such methods is well understood in the case of moderately bounded $H(t)[6,7]$, no results are so far available when $\|H(t)\|$ becomes large. The present paper gives optimal-order estimates for situations where the product of the time step $h$ with $\|H(t)\|$ can be of arbitrary size. Even more interesting than the error bounds themselves are the mechanisms which lead to these bounds and which make Magnus methods perform so well for Schrödinger equations, as compared to standard explicit or implicit numerical integrators.

In Section 2 we recall the concepts underlying the construction of Magnus integrators. Section 3 states the main results, which give asymptotically sharp error bounds for Magnus integrators, in a framework that applies to time-dependent Schrödinger equations requiring neither smallness nor bounds of $h\|H(t)\|$. The general procedure for obtaining such estimates is outlined in Section 4, and is carried out in detail in Sections 5 and 6 for methods of order 2 and 4, respectively. The extension to methods of arbitrary order is done in Section 7. Numerical experiments illustrating the theoretical results are given in Section 8. A basic assumption for the results of this paper are commutator bounds. Their validity for a spectral discretization is shown in the Appendix.

Magnus integrators require computing a matrix exponential multiplying a vector in every time step. For the large matrices (or rather, operators of large dimension) arising from the spatial discretization of Schrödinger equations, this can be done efficiently using operator splitting or Chebyshev or Lanczos approximations. These techniques are well documented in the literature and are not considered here. Because

*Mathematisches Institut, Heinrich-Heine Universität Düsseldorf, Universitätsstr. 1, D-40225 Düsseldorf, Germany (E-mail: marlis@am.uni-duesseldorf.de)

** Mathematisches Institut, Universität Tübingen, Auf der Morgenstelle 10, D-72076 Tübingen, Germany (E-mail: lubich@na.uni-tuebingen.de) 
of the stable error propagation, errors arising from the approximation of the matrix exponentials could be straightforwardly included in the error analysis.

2. Magnus integrators. For the linear differential equation

$$
\dot{y}=A(t) y, \quad y(0)=y_{0},
$$

with a time-dependent matrix $A(t)$, the approach of Magnus [9] aims at writing the solution as

$$
y(t)=\exp (\Omega(t)) y_{0}
$$

for a suitable matrix $\Omega(t)$. An expression for $\Omega(t)$ is obtained by making the ansatz (2.2) and differentiating. This gives

$$
\dot{y}(t)=\operatorname{dexp}_{\Omega(t)}(\dot{\Omega}(t)) y(t),
$$

where the dexp operator can be expressed as

$$
\operatorname{dexp}_{\Omega}(B)=\varphi\left(\operatorname{ad}_{\Omega}\right)(B)=\sum_{k \geq 0} \frac{1}{(k+1) !} \operatorname{ad}_{\Omega}^{k}(B)
$$

with $\varphi(z)=\left(e^{z}-1\right) / z$ and $\operatorname{ad}_{\Omega}(B)=[\Omega, B]=\Omega B-B \Omega$. Hence, (2.2) solves (2.1) if

$$
A(t)=\operatorname{dexp}_{\Omega(t)}(\dot{\Omega}(t)), \quad \Omega(0)=0 .
$$

As long as $\|\Omega(t)\|<\pi$ (which is not the situation of interest in this article!), the operator $\operatorname{dexp} \Omega_{\Omega(t)}$ is invertible and the series

$$
\operatorname{dexp}_{\Omega(t)}^{-1}(A(t))=\sum_{k \geq 0} \frac{\beta_{k}}{k !} \operatorname{ad}_{\Omega(t)}^{k}(A(t))
$$

converges. Here $\beta_{k}$ is the $k$ th Bernoulli number appearing in the series $z /\left(e^{z}-1\right)=$ $\sum_{0}^{\infty}\left(\beta_{k} / k !\right) z^{k}$, which converges for $|z|<2 \pi$. $\left(\right.$ Note $\left\|\operatorname{ad}_{\Omega}(B)\right\| \leq 2\|\Omega\| \cdot\|B\|$, which shows that $(2.5)$ indeed converges for $\|\Omega(t)\|<\pi$.) This gives an explicit differential equation for $\Omega(t)$ :

$$
\dot{\Omega}=A(t)-\frac{1}{2}[\Omega, A(t)]+\frac{1}{12}[\Omega,[\Omega, A(t)]]+\ldots .
$$

Picard iteration yields the Magnus expansion

$$
\begin{aligned}
\Omega(t)=\int_{0}^{t} A(\tau) d \tau & -\frac{1}{2} \int_{0}^{t}\left[\int_{0}^{\tau} A(\sigma) d \sigma, A(\tau)\right] d \tau \\
& +\frac{1}{4} \int_{0}^{t}\left[\int_{0}^{\tau}\left[\int_{0}^{\sigma} A(\mu) d \mu, A(\sigma)\right] d \sigma, A(\tau)\right] d \tau \\
& +\frac{1}{12} \int_{0}^{t}\left[\int_{0}^{\tau} A(\sigma) d \sigma,\left[\int_{0}^{\tau} A(\mu) d \mu, A(\tau)\right]\right] d \tau+\ldots .
\end{aligned}
$$

Numerical methods based on this expansion are reviewed by Iserles, Munthe-Kaas, Nørsett and Zanna [6]. They are of the form

$$
y_{n+1}=\exp \left(\Omega_{n}\right) y_{n}
$$


to give an approximation to $y\left(t_{n+1}\right)$ at $t_{n+1}=t_{n}+h$. Here $\Omega_{n}$ is a suitable approximation of $\Omega(h)$ given by (2.6) with $A\left(t_{n}+\tau\right)$ instead of $A(\tau)$. This approximation first involves truncating the expansion, and second approximating the integrals, e.g., by replacing $A(t)$ locally by an interpolation polynomial $\widehat{A}(t)$ for the nodes $t_{n}+c_{j} h$, so that the integrals in the Magnus expansion can be computed analytically. If $\Omega_{n}$ is built up in this way, then we speak of an interpolatory Magnus integrator. A method of order $p$ is obtained by combining a $p$ th order truncation of the Magnus series and interpolation of $A(t)$ at the nodes of a $p$ th order quadrature formula. A natural choice is Gaussian quadrature.

For example, the midpoint rule yields a second-order scheme with

$$
\Omega_{n}=h A\left(t_{n}+h / 2\right) .
$$

The two-point Gauss quadrature rule has nodes $c_{1,2}=1 / 2 \mp \sqrt{3} / 6$. This yields a fourth-order scheme with

$$
\Omega_{n}=\frac{h}{2}\left(A_{1}+A_{2}\right)+\frac{\sqrt{3} h^{2}}{12}\left[A_{2}, A_{1}\right],
$$

where $A_{j}=A\left(t_{n}+c_{j} h\right), j=1,2$.

High-order interpolatory Magnus integrators require the computation of many commutators per step. Their number can be significantly reduced in specially constructed (non-interpolatory) Magnus integrators as given by Blanes, Casas and Ros [2].

For the purpose of this paper, the Magnus series approach is described only for motivation, since we are interested in the case of large $\|h A(t)\|$, for which $\operatorname{dexp} \Omega_{n}$ need not be invertible and the Magnus expansion need not converge. The known convergence proofs of Magnus series (see [1, 10]) require the time interval to be restricted to $\int_{0}^{t}\|A(\tau)\| d \tau \leq r$ with $r \approx 1$, and there are actually examples of matrix functions with divergent Magnus series for $\int_{0}^{t}\|A(\tau)\| d \tau=\pi$. (The example of $[10$, p. 30] is, admittedly, not of the type studied in this paper.) In any case, the question of convergence of the Magnus series is irrelevant for the problem of obtaining error bounds, much in the same way as the possible convergence or divergence of Taylor series is of no importance for finite-order error bounds elsewhere in numerical analysis. The possible non-invertibility of the dexp operator and even non-existence of a representation (2.2) of the exact solution would appear to be more serious obstacles, but we will show how this problem can be circumvented, using a modified differential equation satisfied by the approximate solution instead of estimating directly the difference between the Magnus expansion and its truncation.

The results of Iserles and Nørsett [7] on the order of Magnus integrators are for $\|h A(t)\| \rightarrow 0$ and are obtained by studying the remainder of the truncated Magnus series (2.6). The constants in those estimates depend on norms of commutators of $A(t)$ for different values of $t$, which all become large with growing $\|A(t)\|$. Therefore, results on the classical order of a method must be taken with caution in the case of the Schrödinger equation, which involves discretizations of unbounded operators. Nevertheless, Magnus integrators work extremely well even with step sizes for which $\|h A(t)\|$ is large. The aim of the present paper is to explain this unexpectedly good behavior.

3. Statement of results. In this section we state our assumptions and main results. Throughout the paper, $\|\cdot\|$ is the Euclidean norm or its induced matrix norm, or occasionally the $L^{2}$ norm of functions. We write

$$
A(t)=-i H(t)=-i(U+V(t)) .
$$


We assume, once and for all, that the hermitian matrix-valued function $V(t)$ and its time derivatives are bounded by

$$
\left\|\frac{d^{m}}{d t^{m}} V(t)\right\| \leq M_{m}, \quad m=0,1,2, \ldots .
$$

The matrix $U$ is assumed symmetric positive definite, with $\|v\| \leq\|U v\|$ for all $v$, but no bound is assumed for the operator norm $\|U\|$. We set

$$
D=U^{1 / 2} \text {. }
$$

The typical situation is given by a discretization of the spatially continuous case where $U=-\Delta+I$, e.g., with periodic boundary conditions on a cube $Q$, and $V(t)$ is a bounded multiplication operator, i.e., $(V(t) v)(x)=V(x, t) v(x)$ for a real-valued smooth potential $V(x, t)$. In this continuous case we have

$$
\|D v\|^{2}=\int_{Q}|\nabla v|^{2} d x+\int_{Q} v^{2} d x,
$$

so that $\|D v\|$ is the familiar $H^{1}$ Sobolev norm of $v$. In the spatially discretized case, $\|D v\|$ can be viewed as a discrete Sobolev norm. For a space discretization with minimal grid spacing $\Delta x$, we note $\|U\| \sim \Delta x^{-2}$ and $\|D\| \sim \Delta x^{-1}$.

Our main assumptions are commutator bounds such as

$$
\|[U, V(t)] v\| \leq K_{0}\|D v\| \quad \text { and } \quad\|[U, \dot{V}(t)] v\| \leq K_{1}\|D v\|
$$

for all $t$ and all vectors $v$. Condition (3.4) is easily verified in the spatially continuous case, with $U=-\Delta+I$ and a smooth potential $V(x, t)$ acting as a multiplication operator. The bound is obtained by noting that in one space dimension, with ${ }^{\prime}=d / d x$,

$$
[U, V] v=-\left((V v)^{\prime \prime}-V v^{\prime \prime}\right)=-\left(2 V^{\prime} v^{\prime}+V^{\prime \prime} v\right)
$$

with the obvious generalization to higher space dimensions. Hence, $[U, V]$ is a firstorder differential operator, which yields (3.4). For a spectral discretization the bound (3.4) is shown, uniformly in the discretization parameter, in [8, Lemma 3.1].

Since $[A(\tau), A(\sigma)]=[U, V(\sigma)-V(\tau)]=\int_{\tau}^{\sigma}[U, \dot{V}(t)] d t$ (when $V(\sigma)$ and $V(\tau)$ commute), the second bound of (3.4) implies, for all vectors $v$,

$$
\|[A(\tau), A(\sigma)] v\| \leq K_{1} h\|D v\| \quad \text { for }|\tau-\sigma| \leq h .
$$

THEOREM 3.1. If $A(t)$ satisfies the commutator bound (3.5), then the error of the exponential midpoint rule (2.7) with (2.8) is bounded by

$$
\left\|y_{n}-y\left(t_{n}\right)\right\| \leq C h^{2} t_{n} \max _{0 \leq t \leq t_{n}}\|D y(t)\|
$$

The constant $C$ depends only on $M_{m}$ for $m \leq 2$ and on $K_{1}$. In particular, $C$ is independent of $n, h$, and $\|D\|$.

This error bound is to be contrasted with the error bound of the classical implicit midpoint rule $y_{n+1}=y_{n}+h A\left(t_{n+1 / 2}\right)\left(y_{n}+y_{n+1}\right) / 2$, for which

$$
\left\|y_{n}-y\left(t_{n}\right)\right\| \leq C h^{2} t_{n} \max _{0 \leq t \leq t_{n}}\left\|\frac{d^{3}}{d t^{3}} y(t)\right\|
$$


Since solutions of Schrödinger equations are in general highly oscillatory, the appearance of higher time derivatives is unfavorable. On the other hand, $\|D y(t)\|^{2}$ represents essentially the quantum kinetic energy, which is bounded a priori. We remark that a similar, but weaker estimate for the exponential midpoint rule, with $\left\|D^{2} y(t)\right\|$ instead of $\|D y(t)\|$, was previously obtained in our paper [5] with a different proof. (That paper unluckily also states an error bound for a third-order Magnus method, without detailed proof and involving the operator norm $\|U\|$, which is superseded by the results of the present paper.)

For methods of order $p$ which contain commutator products of $A\left(t_{n}+c_{j} h\right)$ with $r$ factors (in Example 2 we have $r=2$ for order $p=4$, and $r \leq p-1$ holds for all Magnus methods proposed in the literature), we assume that $A$ satisfies, for all $\tau_{j}$,

$$
\left\|\left[A\left(\tau_{k}\right),\left[\ldots,\left[A\left(\tau_{1}\right), \frac{d^{m}}{d t^{m}} V\left(\tau_{0}\right)\right]\right] \ldots\right] v\right\| \leq K\left\|D^{k} v\right\| \quad\left\{\begin{array}{l}
0 \leq m \leq p \\
k+1 \leq r p .
\end{array}\right.
$$

Like (3.5), condition (3.6) is easily verified in the spatially continuous case. For a spectral space discretization of a time-dependent Schrödinger equation, we show in the Appendix that (3.6) is indeed satisfied uniformly in the discretization parameter. Since $\left[A\left(\tau_{1}\right), A\left(\tau_{0}\right)\right]=\left[A\left(\tau_{1}\right), A\left(\tau_{0}\right)-A\left(\tau_{1}\right)\right]=\left[A\left(\tau_{1}\right), i \int_{\tau_{0}}^{\tau_{1}} \dot{V}(\tau) d \tau\right]$, condition $(3.6)$ implies, whenever $\left|\tau_{1}-\tau_{0}\right| \leq h$,

$$
\left\|\left[A\left(\tau_{k}\right),\left[\ldots,\left[A\left(\tau_{1}\right), A\left(\tau_{0}\right)\right]\right] \ldots\right] v\right\| \leq K h\left\|D^{k} v\right\|, \quad k+1 \leq r p .
$$

Unlike the case of the exponential midpoint rule in Theorem 3.1, convergence of higher-order methods is shown only in the spatially discrete case under a step size restriction

$$
h\|D\| \leq c .
$$

Note that this restriction is milder than the step size restriction for explicit integrators, such as Runge-Kutta methods, for which a more stringent condition $h\|D\|^{2} \leq c$ (i.e., $h\|A(t)\| \leq \gamma$ for some constant $\gamma$ ) is required for stability. The classical error bounds for implicit integrators require smallness of $h\|D\|^{2}$ unless high temporal smoothness is supposed.

The following error bound holds for $p$ th order interpolatory Magnus integrators, i.e., those based on a $p$ th order truncation of the Magnus series and polynomial interpolation of $A(t)$ at the nodes of a $p$ th order quadrature formula (see Section 2).

THEOREM 3.2. If the commutator bounds (3.6) hold, then pth order interpolatory Magnus integrators satisfy the error bound

$$
\left\|y_{n}-y\left(t_{n}\right)\right\| \leq C h^{p} t_{n} \max _{0 \leq t \leq t_{n}}\left\|D^{p-1} y(t)\right\|
$$

for time steps $h$ restricted by (3.8). The constant $C$ depends only on $M_{m}$ for $m \leq p$, on $K, c$, and on $p$. In particular, $C$ is independent of $n, h$, and $\|D\|$ as long as $h\|D\| \leq c$.

The error bound of Theorem 3.2 is valid also for non-interpolatory Magnus methods if the quadrature error satisfies, for all $v$,

$$
\left\|\Omega_{n} v-\widetilde{\Omega}_{n} v\right\| \leq C h^{p+1}\left\|D^{p-1} v\right\|,
$$

where $\widetilde{\Omega}_{n}$ denotes the $p$ th order truncation of the Magnus series at $t_{n}$. This generalization of Theorem 3.2 follows directly from the proof below. 
Condition (3.8) is not required for stability. If such a condition on the step size is not imposed, there is still $p$ th order convergence, though only for much smoother solutions: the error is then bounded by $C_{p} h^{p} \max \left\|D^{p-1} y(t)\right\|+C_{p+1} h^{p+1} \max \left\|D^{p} y(t)\right\|+$ $\cdots+C_{p r} h^{p r} \max \left\|D^{p r-1} y(t)\right\|$.

Though Theorem 3.2 is formulated for arbitrary order $p$, we note that high-order error bounds are of limited value in the approximation of highly oscillatory solutions, for which (discretized) high-order derivatives $D^{k} y(t)$ have progressively much larger norms.

Theorems 3.1 and 3.2 are proved in the remainder of this article. In the following section we describe a general procedure for deriving error bounds. We will follow this procedure in detail for the exponential midpoint rule in Section 5 and for fourth-order methods in Section 6. This gives all the tools for the extension to the general case, which is treated in Section 7 .

4. General procedure for deriving error bounds. The convergence analysis is done in two steps. In the first step we study the error which results from truncating the Magnus expansion; in the second step, we discuss the error resulting from approximating the integrals by quadrature. (In the estimates of this and the following sections, $C$ is a generic constant which assumes different values on different occurrences.)

Truncation of the Magnus expansion amounts to using a modified $\widetilde{\Omega}$ instead of $\Omega$ in $(2.2)$, i.e.

$$
\widetilde{y}(t)=\exp (\widetilde{\Omega}(t)) y_{0}
$$

By differentiating, we obtain the approximate solution $\widetilde{y}(t)$ as the solution of the modified differential equation

$$
\dot{\widetilde{y}}(t)=\widetilde{A}(t) \widetilde{y}(t) \quad \text { with } \quad \widetilde{A}(t)=\operatorname{dexp}_{\widetilde{\Omega}(t)}(\dot{\widetilde{\Omega}}(t))
$$

with initial value $\widetilde{y}(0)=y_{0}$. Note that the truncated Magnus series $\widetilde{\Omega}(t)$ and the modified operator $\widetilde{A}(t)$ are skew hermitian if $A(t)$ is skew hermitian. As the following lemma shows, a bound on $\widetilde{A}-A$ then immediately gives a local error bound.

LEMMA 4.1. Let $y$ be a solution of (2.1) with skew-hermitian $A, \widetilde{y}$ a solution of (4.1). With $E=\widetilde{A}-A$, the error satisfies

$$
\|\widetilde{y}(t)-y(t)\| \leq \int_{0}^{t}\|E(\tau) y(\tau)\| d \tau .
$$

Proof. We write (2.1) as $\dot{y}=A(t) y=\widetilde{A}(t) y-E(t) y$ and subtract (4.1). This shows that the error $\widetilde{\varepsilon}=\widetilde{y}-y$ satisfies

$$
\dot{\widetilde{\varepsilon}}=\widetilde{A}(t) \widetilde{\varepsilon}+E(t) y, \quad \widetilde{\varepsilon}(0)=0 .
$$

Since $\widetilde{A}$ is skew hermitian, taking the inner product with $\widetilde{\varepsilon}$ on both sides leads to

$$
\langle\dot{\widetilde{\varepsilon}}, \widetilde{\varepsilon}\rangle=\langle E y, \widetilde{\varepsilon}\rangle \leq\|E y\|\|\widetilde{\varepsilon}\| \text {. }
$$

On the other hand, $\langle\dot{\widetilde{\varepsilon}}, \widetilde{\varepsilon}\rangle=\frac{1}{2} \frac{d}{d t}\|\widetilde{\varepsilon}\|^{2}=\frac{d}{d t}\|\widetilde{\varepsilon}\| \cdot\|\widetilde{\varepsilon}\|$. Integrating the inequality proves the lemma. 
A crucial step in obtaining a bound on $E=\widetilde{A}-A$ is truncating the dexp series (2.3) and providing a bound for the remainder. We define the remainder function $r_{p}$, for $p \geq 1$, via

$$
\frac{e^{z}-1}{z}=1+\frac{1}{2} z+\ldots+\frac{1}{(p-1) !} z^{p-2}+\frac{1}{p !} z^{p-1} r_{p}(z)
$$

so that

$$
\operatorname{dexp}_{\Omega}(B)=B+\frac{1}{2}[\Omega, B]+\ldots+\frac{1}{(p-1) !} \operatorname{ad}_{\Omega}^{p-2}(B)+\frac{1}{p !} r_{p}\left(\operatorname{ad}_{\Omega}\right)\left(\operatorname{ad}_{\Omega}^{p-1}(B)\right) .
$$

For $A(t)$ of the form (3.1) satisfying the conditions of Section 3, we will bound the remainder term by

$$
\left\|r_{p}\left(\operatorname{ad}_{\widetilde{\Omega}(t)}\right)\left(\operatorname{ad}_{\widetilde{\Omega}(t)}^{p-1}(\dot{\widetilde{\Omega}}(t))\right) v\right\| \leq C h^{p}\left\|D^{p-1} v\right\|, \quad 0 \leq t \leq h .
$$

In the case of $p>2$, it turns out that the bound requires time steps $h$ with (3.8), while for $p=2$, no restriction on $h$ is necessary.

Next we incorporate the error resulting from approximating the integrals. In the $n$th time step, we take $\widetilde{\Omega}(h)$ corresponding to the truncated Magnus series for $A\left(t_{n}+t\right)$ instead of $A(t)$, which we denote by $\widetilde{\Omega}_{n}$. By the quadrature approximation, $\widetilde{\Omega}_{n}$ is replaced by $\Omega_{n}$ with which the actual computations are done. This approximation typically satisfies

$$
\left\|\left(\widetilde{\Omega}_{n}-\Omega_{n}\right) v\right\| \leq C h^{p+1}\left\|D^{r-1} v\right\|
$$

where $p$ is the order of the quadrature rule and commutator products with $r$ factors appear in the method. For the exponential midpoint rule $(p=2, r=1)$ this bound is independent of $D$. For $p$ th-order interpolatory Magnus schemes (where $r \leq p-1$ ) we will show that (4.5) holds and that this leads to the local error bound

$$
\left\|\exp \left(\widetilde{\Omega}_{n}\right) v-\exp \left(\Omega_{n}\right) v\right\| \leq C h^{p+1}\left\|D^{r-1} v\right\| .
$$

Putting both steps together, the exact solution $y$ of (2.1) satisfies

$$
y\left(t_{n+1}\right)=\exp \left(\Omega_{n}\right) y\left(t_{n}\right)+\varepsilon_{n},
$$

with $\varepsilon_{n}=y\left(t_{n+1}\right)-\exp \left(\widetilde{\Omega}_{n}\right) y\left(t_{n}\right)+\exp \left(\widetilde{\Omega}_{n}\right) y\left(t_{n}\right)-\exp \left(\Omega_{n}\right) y\left(t_{n}\right)$. By Lemma 4.1 and (4.6), this gives

$$
\left\|\varepsilon_{n}\right\| \leq \int_{t_{n}}^{t_{n+1}}\|E(\tau) y(\tau)\| d \tau+C h^{p+1}\left\|D^{r-1} y\left(t_{n}\right)\right\| .
$$

Subtracting (2.7) from (4.7) leads to the error recursion for $e_{n}=y_{n}-y\left(t_{n}\right)$ :

$$
e_{n+1}=\exp \left(\Omega_{n}\right) e_{n}+\varepsilon_{n},
$$

and thus

$$
\left\|e_{n}\right\| \leq \sum_{j=0}^{n-1}\left\|\varepsilon_{j}\right\|
$$

In summary, error bounds for general Magnus methods are obtained as follows: We have to provide a bound on $E(t) y(t)$, which basically means to prove (4.4), and we have to show that the approximation $\Omega_{n}$ satisfies (4.6). This program is carried out in the following sections. 
5. Error bounds for the exponential midpoint rule. In this section we prove Theorem 3.1. The second order truncation of the Magnus expansion is simply

$$
\widetilde{\Omega}(t)=\int_{0}^{t} A(\tau) d \tau, \quad 0 \leq t \leq h .
$$

Following the approach described in Section 4 , we know that $\widetilde{y}(t)=\exp (\widetilde{\Omega}(t)) y_{0}$ solves (4.1) with

$$
\widetilde{A}(t)=\operatorname{dexp}_{\widetilde{\Omega}(t)}(\dot{\widetilde{\Omega}}(t))=A(t)+\frac{1}{2} r_{2}\left(\operatorname{ad}_{\widetilde{\Omega}(t)}\right)\left(\operatorname{ad}_{\widetilde{\Omega}(t)}(\dot{\widetilde{\Omega}}(t))\right)=: A(t)+E_{2}(t)
$$

where the representation (4.3) for the dexp operator was used. The remainder $r_{2}$ was defined in (4.2).

Lemma 5.1. $r_{2}$ satisfies (4.4) with $p=2$, where the constant $C$ depends only on $M_{0}$ of (3.2) and $K_{1}$ of (3.5).

Proof. (a) We fix an arbitrary $t$ with $0 \leq t \leq h$. After an orthogonal similarity transform, we may assume that $\Omega:=\widetilde{\Omega}(t)$ is diagonal, $\Omega=\operatorname{diag}\left(\omega_{k}\right)$ with purely imaginary eigenvalues $\omega_{k}$, and we define $B=\dot{\widetilde{\Omega}}(t)$. Denoting by $\bullet$ the entrywise product of matrices, we can write

$$
\operatorname{ad}_{\Omega}(B)=\Omega B-B \Omega=Z \bullet B,
$$

where $Z=\left(\omega_{k}-\omega_{\ell}\right)_{k, \ell}$. This yields

$$
r_{p}\left(\operatorname{ad}_{\Omega}\right)\left(\operatorname{ad}_{\Omega}^{p-1}(B)\right) v=\left(R \bullet \operatorname{ad}_{\Omega}^{p-1}(B)\right) v,
$$

where $R=\left(r_{p}\left(\omega_{k}-\omega_{\ell}\right)\right)_{k, \ell}$. We now follow the proof of Lemma 2.2 of [5]. Note that for real $x, r_{p}(i x)=1+O(x), x \rightarrow 0$ and $r_{p}(i x)=O\left(x^{-1}\right),|x| \rightarrow \infty$ and hence $r_{p}, r_{p}^{\prime} \in L^{2}(i \mathbb{R})$. As can be seen, e.g., from formula (2.13) in [5], $r_{p}$ has a Fourier transform $\widehat{r}_{p} \in L^{1}(\mathbb{R})$,

$$
r_{p}(i x)=\int_{\mathbb{R}} e^{i \xi x} \widehat{r}_{p}(\xi) d \xi,
$$

with $\left\|\widehat{r}_{p}\right\|_{L^{1}(\mathbb{R})} \leq 2 \pi\left\|r_{p}\right\|_{L^{2}(i \mathbb{R})}^{1 / 2}\left\|r_{p}^{\prime}\right\|_{L^{2}(i \mathbb{R})}^{1 / 2}$. Consequently, the above expression can be written as

$$
r_{p}\left(\operatorname{ad}_{\Omega}\right)\left(\operatorname{ad}_{\Omega}^{p-1}(B)\right) v=\int_{\mathbb{R}} \widehat{r}_{p}(\xi) \exp (\xi \Omega) \operatorname{ad}_{\Omega}^{p-1}(B) \exp (-\xi \Omega) v d \xi,
$$

so that

$$
\left\|r_{p}\left(\operatorname{ad}_{\Omega}\right)\left(\operatorname{ad}_{\Omega}^{p-1}(B)\right) v\right\| \leq\left\|\widehat{r}_{p}\right\|_{L^{1}(\mathbb{R})} \sup _{\xi \in \mathbb{R}}\left\|\operatorname{ad}_{\Omega}^{p-1}(B) \exp (-\xi \Omega) v\right\| .
$$

So far, this holds for general $p$.

(b) From now on we set $p=2$. Using

$$
\operatorname{ad}_{\Omega}(B)=\operatorname{ad}_{\widetilde{\Omega}(t)}(\dot{\widetilde{\Omega}}(t))=[\widetilde{\Omega}(t), \dot{\widetilde{\Omega}}(t)]=\int_{0}^{t}[A(\tau), A(t)] d \tau
$$


and (3.5), we obtain for all vectors $w$

$$
\left\|\operatorname{ad}_{\Omega}(B) w\right\| \leq K_{1} h^{2}\|D w\|
$$

Hence we have

$$
\left\|r_{p}\left(\operatorname{ad}_{\Omega}\right)\left(\operatorname{ad}_{\Omega}^{p-1}(B)\right) v\right\| \leq C h^{2} \sup _{\xi \in \mathbb{R}}\|D \exp (-\xi \Omega) v\|
$$

We now use the splitting (3.1) and write

$$
\frac{i}{t} \Omega=U+\frac{1}{t} \int_{0}^{t} V(\tau) d \tau=: U+\widetilde{V} .
$$

We choose $\alpha \geq 0$ such that $U+\widetilde{V}+\alpha I$ is symmetric and positive definite. To keep the notation simple, we omit the constants and denote by $\sim$ equivalent norms. Because of the boundedness of $\widetilde{V}$ and (3.3) we have for all vectors $w$

$$
\|D w\|=\sqrt{w^{*} U w} \sim \sqrt{w^{*}(U+\widetilde{V}+\alpha I) w}=\left\|\left(\frac{i}{t} \Omega+\alpha I\right)^{1 / 2} w\right\| .
$$

We use this norm equivalence to bound the last factor in (5.3):

$$
\begin{aligned}
\|D \exp (-\xi \Omega) v\| & \sim\left\|\left(\frac{i}{t} \Omega+\alpha I\right)^{1 / 2} \exp (-\xi \Omega) v\right\| \\
& =\left\|\exp (-\xi \Omega)\left(\frac{i}{t} \Omega+\alpha I\right)^{1 / 2} v\right\| \\
& =\left\|\left(\frac{i}{t} \Omega+\alpha I\right)^{1 / 2} v\right\| \\
& \sim\|D v\| .
\end{aligned}
$$

Inserting this into (5.3) proves the lemma.

By definition (5.1) of $E_{2}$, this immediately yields the bound

$$
\left\|E_{2}(t) y(t)\right\| \leq C h^{2}\|D y(t)\|, \quad 0 \leq t \leq h .
$$

Applying Lemma 4.1 gives

$$
\|\widetilde{\varepsilon}(t)\| \leq C h^{3} \max _{0 \leq \tau \leq h}\|D y(\tau)\|
$$

The midpoint rule uses the approximation

$$
\widetilde{\Omega}_{n}=\int_{0}^{h} A\left(t_{n}+\tau\right) d \tau \approx h A\left(t_{n+1 / 2}\right)=: \Omega_{n}
$$

in the scheme (2.7). The midpoint rule is of order two, and since $\|\ddot{A}(t)\| \leq M_{2}$, the quadrature error is bounded by

$$
\left\|\widetilde{\Omega}_{n}-\Omega_{n}\right\| \leq \frac{1}{24} M_{2} h^{3} .
$$

The identity

$$
\exp \left(\widetilde{\Omega}_{n}\right)-\exp \left(\Omega_{n}\right)=\int_{0}^{1} \exp \left((1-s) \Omega_{n}\right)\left(\widetilde{\Omega}_{n}-\Omega_{n}\right) \exp \left(s \widetilde{\Omega}_{n}\right) d s
$$


then yields

$$
\left\|\exp \left(\widetilde{\Omega}_{n}\right)-\exp \left(\Omega_{n}\right)\right\| \leq \frac{1}{24} M_{2} h^{3} .
$$

Combining (5.4) and (5.5) yields for the defects $\varepsilon_{j}$ of (4.7)

$$
\left\|\varepsilon_{j}\right\| \leq C h^{3} \max _{t_{j} \leq \tau \leq t_{j+1}}\|D y(\tau)\| .
$$

By (4.8), this gives

$$
\left\|e_{n}\right\| \leq C h^{2} t_{n} \max _{0 \leq t \leq t_{n}}\|D y(t)\|
$$

which is just the statement of Theorem 3.1.

6. Error bounds for fourth order Magnus methods. This section gives the proof of Theorem 3.2 for $p=4$. It provides all the machinery needed for treating general order $p$, but still gives an explicit presentation of the appearing terms.

A Magnus method of classical order four is constructed by setting

$$
\dot{\widetilde{\Omega}}(t)=A\left(t_{n}+t\right)-\frac{1}{2} \int_{0}^{t}\left[A\left(t_{n}+\tau\right), A\left(t_{n}+t\right)\right] d \tau, \quad \widetilde{\Omega}\left(t_{n}\right)=0
$$

for $0 \leq t \leq h$. To study the local error we simplify the notation and consider the case $n=0$. Then integration yields

$$
\widetilde{\Omega}(t)=\int_{0}^{t} A(\tau) d \tau-\frac{1}{2} \int_{0}^{t} \int_{0}^{\tau}[A(\sigma), A(\tau)] d \sigma d \tau, \quad 0 \leq t \leq h .
$$

In this case, $\widetilde{y}(t)=\exp (\widetilde{\Omega}(t)) y_{0}$ solves (4.1) with (partly omitting the argument $t$ )

$$
\widetilde{A}(t)=\dot{\widetilde{\Omega}}(t)+\frac{1}{2}[\widetilde{\Omega}, \dot{\widetilde{\Omega}}]+\frac{1}{6}[\widetilde{\Omega},[\widetilde{\Omega}, \dot{\widetilde{\Omega}}]]+\frac{1}{24} r_{4}\left(\operatorname{ad}_{\tilde{\Omega}}\right)\left(\operatorname{ad}_{\widetilde{\Omega}}^{3}(\dot{\widetilde{\Omega}})\right)
$$

Lemma 6.1. If $h\|D\| \leq c$, then $r_{4}$ defined in (4.2) satisfies (4.4) with $p=4$, where the constant $C$ depends only on $K, M_{0}$, and $c$.

Proof. (a) The first part of the proof is identical to part (a) of the proof of Lemma 5.1. We write again, for fixed $t$ with $0 \leq t \leq h, \Omega=\widetilde{\Omega}(t)$ and $B=\dot{\widetilde{\Omega}}(t)$, for $\widetilde{\Omega}(t)$ of (6.2). We start with the bound (5.2) and turn to estimate $\operatorname{ad}_{\Omega}^{3}(B) w$. Using the commutator bound (3.7) (and previously the Jacobi identity, if necessary) for terms such as, e.g.,

$$
\left\|\left[\int_{0}^{t} A(\tau) d \tau,\left[\int_{0}^{t} A(\tau) d \tau,\left[\int_{0}^{t} \int_{0}^{\tau}[A(\sigma), A(\tau)] d \sigma d \tau, A(t)\right]\right]\right] w\right\| \leq K h^{5}\left\|D^{4} w\right\|,
$$

it is shown under the restriction $h\|D\| \leq c$ that for all $w$,

$$
\left\|\operatorname{ad}_{\Omega}^{3}(B) w\right\| \leq C h^{4}\left\|D^{3} w\right\|, \quad 0 \leq t \leq h .
$$

Inserted in (5.2), this bound yields

$$
r_{4}\left(\operatorname{ad}_{\Omega}\right)\left(\operatorname{ad}_{\Omega}^{3}(B)\right) v \leq C h^{4} \sup _{\xi \in \mathbb{R}}\left\|D^{3} \exp (-\xi \Omega) v\right\| .
$$


(b) It remains to show that the supremum can be bounded by $C\left\|D^{3} v\right\|$. We use the splitting (3.1) and write, still for fixed $t$ with $0<t \leq h$,

$$
\frac{i}{t} \Omega=U+\frac{1}{t} \int_{0}^{t} V(\tau) d \tau-\frac{i}{2 t} \int_{0}^{t} \int_{0}^{\tau}[A(\sigma), A(\tau)] d \sigma d \tau=: U+\tilde{V} .
$$

By the assumptions, $\widetilde{V}=V(0)+O(h)$ is a hermitian bounded operator, and thus there exists $\alpha \geq 0$ such that $U+\widetilde{V}+\alpha I$ is positive definite. Our next aim is to show that for all $w$,

$$
\left\|D^{4} w\right\|=\left\|U^{2} w\right\| \sim\left\|\left(\frac{i}{t} \Omega+\alpha I\right)^{2} w\right\|
$$

We have

$$
(U+\widetilde{V}+\alpha I)^{2}-U^{2}=2(\widetilde{V}+\alpha I) U+[U, \tilde{V}+\alpha I]+(\tilde{V}+\alpha I)^{2} .
$$

The first and the last term on the right-hand side yield bounds

$$
\|2(\widetilde{V}+\alpha I) U w\|+\left\|(\widetilde{V}+\alpha I)^{2} w\right\| \leq C\left\|D^{2} w\right\|+C\|w\|
$$

Bounds for the second term are obtained from assumption (3.7). By definition of $\widetilde{V}$ and writing $U=i A(\tau)-V(\tau)$, we have

$$
\begin{aligned}
{[U, \widetilde{V}]=} & {\left[U, \frac{1}{t} \int_{0}^{t} V(\tau) d \tau-\frac{i}{2 t} \int_{0}^{t} \int_{0}^{\tau}[A(\sigma), A(\tau)] d \sigma d \tau\right] } \\
= & \frac{i}{t} \int_{0}^{t}[A(\tau), V(\tau)] d \tau \\
& +\frac{1}{2 t} \int_{0}^{t} \int_{0}^{\tau}[A(0),[A(\sigma), A(\tau)]] d \sigma d \tau \\
& +\frac{i}{2 t} \int_{0}^{t} \int_{0}^{\tau}[V(0),[A(\sigma), A(\tau)]] d \sigma d \tau .
\end{aligned}
$$

By the commutator bounds (3.6) and (3.7) and the Jacobi identity, we obtain for $h\|D\| \leq c$

$$
\|[U, \widetilde{V}] w\| \leq K\|D w\|+\frac{1}{2} K h^{2}\left\|D^{2} w\right\|+K h\left\|D^{2} w\right\| \leq C\|D w\| .
$$

Together with (6.6) this proves (6.5). Moreover, the estimates (6.6) and (6.7) show that

$$
\left\|\left((U+\widetilde{V}+\alpha I)^{2}-U^{2}\right) U^{-1} w\right\| \leq C\|w\|
$$

So we can apply Lemma 6.2 below with $\mu=1 / 2$ and $\theta=3 / 4$, to show that $(6.5)$ implies

$$
\left\|D^{3} w\right\|=\left\|U^{3 / 2} w\right\| \sim\left\|\left(\frac{i}{t} \Omega+\alpha I\right)^{3 / 2} w\right\| .
$$

As at the end of the proof of Lemma 5.1, we then obtain

$$
\left\|D^{3} \exp (-\xi \Omega) v\right\| \leq C\left\|D^{3} v\right\|
$$


with a constant independent of $\xi \in \mathbb{R}$ and $t$ with $0<t \leq h$. Inserting this bound in (6.4) completes the proof.

LEMMA 6.2. Suppose $S, T$ are hermitian positive definite operators such that $\left\|(S-T) S^{-\mu}\right\| \leq M$ holds with $0 \leq \mu<1$. If

$$
\|S v\| \leq\|T v\| \quad \text { for all } v
$$

then, for $0<\theta<1$,

$$
\left\|S^{\theta} v\right\| \leq C\left\|T^{\theta} v\right\| \quad \text { for all } v
$$

where $C$ depends only on $M$ and $\mu$.

Proof. This is a reformulation of Theorem 1.4.6 in [4].

We are now in the position to prove a bound of $\widetilde{A}(t)-A(t)$.

Lemma 6.3. For $\widetilde{A}(t)$ defined in (6.3) and time steps $h$ with $h\|D\| \leq c$, the error $E_{4}(t):=\widetilde{A}(t)-A(t)$ is bounded, for all vectors $v$, by

$$
\left\|E_{4}(t) v\right\| \leq C h^{4}\left\|D^{3} v\right\|, \quad 0 \leq t \leq h .
$$

The constant $C$ depends only on $K, M_{0}, M_{1}$, and $c$.

Proof. We insert (6.1) and (6.2) into (6.3):

$$
\begin{aligned}
E_{4}(t)= & -\frac{1}{12} \int_{0}^{t} \int_{0}^{t} \int_{0}^{t}[A(\mu),[A(\tau),[A(\sigma), A(t)]]] d \sigma d \tau d \mu \\
& -\frac{1}{12} \int_{0}^{t} \int_{0}^{t} \int_{0}^{\tau}[A(\mu),[[A(\sigma), A(\tau)], A(t)]] d \sigma d \tau d \mu \\
& +\frac{1}{24} \int_{0}^{t} \int_{0}^{\mu} \int_{0}^{t}[[A(\sigma), A(\mu)],[A(\tau), A(t)]] d \tau d \sigma d \mu+R(t) \\
& +\frac{1}{24} r_{4}\left(\operatorname{ad}_{\tilde{\Omega}}\right)\left(\operatorname{ad}_{\widetilde{\Omega}}^{3}(\dot{\widetilde{\Omega}})\right)
\end{aligned}
$$

Here, $R(t) v$ contains integrals of commutators which, by (3.7), are bounded by

$$
C\left(h^{5}\left\|D^{4} v\right\|+h^{6}\left\|D^{5} v\right\|\right) \leq C^{\prime} h^{4}\left\|D^{3} v\right\|
$$

for $h\|D\| \leq c$. The constant $C$ only depends on $K$. Then, by (3.7),

$$
\left\|E_{4}(t) v\right\| \leq C h^{4}\left\|D^{3} v\right\|+\frac{1}{24}\left\|r_{4}\left(\operatorname{ad}_{\widetilde{\Omega}}\right)\left(\operatorname{ad}_{\widetilde{\Omega}}^{3}(\dot{\widetilde{\Omega}})\right) v\right\| .
$$

The bound (6.8) now follows from Lemma 6.1. $\square$

Lemma 4.1 shows that $\widetilde{\varepsilon}=\widetilde{y}-y$ is bounded by

$$
\|\widetilde{\varepsilon}(t)\| \leq C h^{5} \max _{0 \leq \tau \leq h}\left\|D^{3} y(\tau)\right\|, \quad 0 \leq t \leq h .
$$

Since we want to have a fourth order scheme, we use a quadrature formula $\left(b_{i}, c_{i}\right)_{i=1}^{s}$ of order $p \geq 4$. In (6.2) we replace $A$ by its interpolation polynomial $\widehat{A}$ in the nodes $t_{n}+c_{j} h$. The integrals can then be evaluated exactly. The quadrature error for $n=0$ is given by

$$
\begin{aligned}
\widetilde{\Omega}_{0}-\Omega_{0}= & \int_{0}^{h} A(\tau) d \tau-\int_{0}^{h} \widehat{A}(\tau) d \tau \\
& -\left(\frac{1}{2} \int_{0}^{h} \int_{0}^{\tau}[A(\sigma), A(\tau)] d \sigma d \tau-\frac{1}{2} \int_{0}^{h} \int_{0}^{\tau}[\widehat{A}(\sigma), \widehat{A}(\tau)] d \sigma d \tau\right),
\end{aligned}
$$


and similarly for the general $n$th step with $A\left(t_{n}+\tau\right)$ instead of $A(\tau)$.

LEMma 6.4. The quadrature error in the nth step satisfies

$$
\left\|\left(\widetilde{\Omega}_{n}-\Omega_{n}\right) v\right\| \leq C h^{p+1}\|D v\| .
$$

The constant $C$ depends only on $M_{m}$ for $m \leq p$ and $K_{1}$.

Proof. For ease of notation we let $n=0$. The error of the single integral in the representation of $\widetilde{\Omega}_{n}-\Omega_{n}$ is $O\left(h^{p+1}\right)$. Assume that we use a quadrature rule with $s$ nodes. For estimating the error of the double integral we define the interpolation error

$$
J(t):=A(t)-\widehat{A}(t)=h^{s} \int_{0}^{1} \widehat{\kappa}_{s}(\theta, \vartheta) A^{(s)}(\theta h) d \theta, \quad 0 \leq t=\vartheta h \leq h,
$$

where $\widehat{\kappa}_{s}$ denotes the Peano kernel. The difficulty in the remaining proof comes from the fact that we have only $J(t)=O\left(h^{s}\right)$, but we need an $O\left(h^{p}\right)$ estimate. We use in addition $J\left(c_{i} h\right)=0$ and $\int_{0}^{h} J(t) d t=O\left(h^{p+1}\right)$. For the second term in (6.9) we write

$$
\begin{aligned}
\int_{0}^{h} \int_{0}^{\tau} & {[A(\sigma), A(\tau)] d \sigma d \tau-\int_{0}^{h} \int_{0}^{\tau}[\widehat{A}(\sigma), \widehat{A}(\tau)] d \sigma d \tau } \\
& =\int_{0}^{h} \int_{0}^{\tau}([\widehat{A}(\sigma), J(\tau)]+[J(\sigma), \widehat{A}(\tau)]+[J(\sigma), J(\tau)]) d \sigma d \tau .
\end{aligned}
$$

Approximating the outer integral with the quadrature formula, the first term becomes

$$
\int_{0}^{h} \int_{0}^{\tau}[\widehat{A}(\sigma), J(\tau)] d \sigma d \tau=h^{p+1} \int_{0}^{1} \kappa_{p}(\theta) G^{(p)}(\theta h) d \theta,
$$

where $\kappa_{p}$ is the Peano kernel, and

$$
G(\tau)=\int_{0}^{\tau}[\widehat{A}(\sigma), J(\tau)] d \sigma .
$$

Using Leibniz' rule, it is seen that the dominant term of $G^{(p)}(\tau)$ is $p\left[\widehat{A}(\tau), J^{(p-1)}(\tau)\right]$, so that by (3.6), for any vector $v$,

$$
\left\|G^{(p)}(\theta h) v\right\| \leq C\|D v\| .
$$

This yields

$$
\left\|\int_{0}^{h} \int_{0}^{\tau}[\widehat{A}(\sigma), J(\tau)] d \sigma d \tau v\right\| \leq C h^{p+1}\|D v\| .
$$

For the second term, we use partial integration

$$
\int_{0}^{h} \int_{0}^{\tau}[J(\sigma), \widehat{A}(\tau)] d \sigma d \tau=\left[\int_{0}^{h} J(\sigma) d \sigma, \int_{0}^{h} \widehat{A}(\mu) d \mu\right]-\int_{0}^{h} \int_{0}^{\tau}[J(\tau), \widehat{A}(\sigma)] d \sigma d \tau .
$$

Here, for the last term, the bound was already given in (6.12). Using the quadrature formula for the integral over $J$, we have for the first term

$$
\left[\int_{0}^{h} J(\sigma) d \sigma, \int_{0}^{h} \widehat{A}(\mu) d \mu\right]=h^{p+1}\left[\int_{0}^{1} \kappa_{p}(\theta) J^{(p)}(\theta h) d \theta, \int_{0}^{h} \widehat{A}(\mu) d \mu\right] .
$$


Noting $J^{(p)}(t)=A^{(p)}(t)$ and using (3.6), this gives the bound

$$
\left\|\int_{0}^{h} \int_{0}^{\tau}[J(\sigma), \widehat{A}(\tau)] d \sigma d \tau v\right\| \leq C h^{p+1}\|D v\| .
$$

Finally, since $\|J(t)\|=O\left(h^{s}\right)$,

$$
\left\|\int_{0}^{h} \int_{0}^{\tau}[J(\sigma), J(\tau)] d \sigma d \tau v\right\| \leq C h^{2 s+2}\|v\| \leq C h^{p+2}\|v\| .
$$

Inserting the bounds (6.12)-(6.14) in (6.11) completes the proof.

LEMMA 6.5. In the situation of Lemma 6.4,

$$
\left\|\exp \left(\widetilde{\Omega}_{n}\right) v-\exp \left(\Omega_{n}\right) v\right\| \leq C h^{p+1}\|D v\| .
$$

The constant $C$ depends only on $M_{m}$ for $m \leq p$ and $K_{1}$.

Proof. The variation-of-constants formula yields

$$
\exp \left(\widetilde{\Omega}_{n}\right) v-\exp \left(\Omega_{n}\right) v=\int_{0}^{1} \exp \left((1-s) \Omega_{n}\right)\left(\widetilde{\Omega}_{n}-\Omega_{n}\right) \exp \left(s \widetilde{\Omega}_{n}\right) v d s .
$$

By (6.10) we have

$$
\left\|\left(\widetilde{\Omega}_{n}-\Omega_{n}\right) \exp \left(s \widetilde{\Omega}_{n}\right) v\right\| \leq C h^{p+1}\left\|D \exp \left(s \widetilde{\Omega}_{n}\right) v\right\| \leq C^{\prime} h^{p+1}\|D v\|,
$$

where the last inequality is obtained as in the proof of Lemma 5.1. This gives the stated bound.

For $p \geq 4$, the local error $\varepsilon_{n}$ of the scheme (2.7) thus satisfies (4.7) with

$$
\left\|\varepsilon_{n}\right\| \leq C h^{5} \max _{t_{n} \leq t \leq t_{n+1}}\left\|D^{3} y(t)\right\| .
$$

Hence, with (4.8), the global error is bounded by

$$
\left\|e_{n}\right\| \leq C t_{n} h^{4} \max _{0 \leq t \leq t_{n}}\left\|D^{3} y(t)\right\|,
$$

and Theorem 3.2 is proved for $p=4$.

7. Error bounds for higher-order Magnus integrators. The arguments of the previous section can be extended rather directly to methods of arbitrary order. In the following we describe this extension, putting the emphasis on the general structure and on a few additional considerations that become necessary. Though it would have been possible to present the general proof without first discussing the second and fourth order cases, we believe that it is useful to have seen and understood the explicit expressions arising in the proofs for the lower order methods before embarking on the general case.

Lemma 7.1. If $\|h D\| \leq c$, then $r_{p}$ defined in (4.2) satisfies, for a pth order truncated Magnus expansion $\widetilde{\Omega}(t)$, the bound (4.4) where the constant $C$ depends only on $K, M_{0}, c$, and $p$.

Proof. For the truncated Magnus series $\widetilde{\Omega}(t)$, the expression $\operatorname{ad}_{\widetilde{\Omega}(t)}^{p-1}(\dot{\widetilde{\Omega}}(t))$ consists (after repeated use of the Jacobi identity) of a linear combination of iterated commutators of $A(\cdot)$ integrated over all but one of the independent variables over intervals bounded by $h$. The appearing iterated commutators and integrals are at least 
$(p-1)$-fold. Together with the commutator bound (3.7) and $\|h D\| \leq c$ this yields the bound

$$
\left\|\operatorname{ad}_{\widetilde{\Omega}(t)}^{p-1}(\dot{\widetilde{\Omega}}(t)) w\right\| \leq C h^{p}\left\|D^{p-1} w\right\|
$$

By (5.2), this implies

$$
\left\|r_{p}\left(\operatorname{ad}_{\widetilde{\Omega}(t)}\right) \operatorname{ad}_{\widetilde{\Omega}(t)}^{p-1}(\dot{\widetilde{\Omega}}(t)) v\right\| \leq C h^{p} \sup _{\xi \in \mathbb{R}}\left\|D^{p-1} \exp (\xi \widetilde{\Omega}(t)) v\right\|
$$

for all $v$. By a straightforward, but tedious generalization of the argument in the proof of Lemma 6.1, the supremum is bounded by

$$
\sup _{\xi \in \mathbb{R}}\left\|D^{p-1} \exp (\xi \widetilde{\Omega}(t)) v\right\| \leq C\left\|D^{p-1} v\right\|
$$

which yields the desired bound (4.4).

LEMMA 7.2. For $\widetilde{A}(t)$ defined in (4.1) and time steps $h$ with $h\|D\| \leq c$, the error $E_{p}(t):=\widetilde{A}(t)-A(t)$ is bounded, for all vectors $v$, by

$$
\left\|E_{p}(t) v\right\| \leq C h^{p}\left\|D^{p-1} v\right\|, \quad 0 \leq t \leq h .
$$

The constant $C$ depends only on $K, M_{0}, M_{1}, c$, and $p$.

Proof. By construction of the Magnus series, $E_{p}(t)$ is a linear combination of at least $(p-1)$-fold integrals of iterated commutators of $A(\cdot)$ and $r_{p}\left(\operatorname{ad}_{\widetilde{\Omega}(t)}\right) \operatorname{ad}_{\widetilde{\Omega}(t)}^{p-1}(\dot{\widetilde{\Omega}}(t))$. The stated estimate thus follows from the commutator bound (3.7), the step size bound (3.8), and Lemma 7.1.

Consider a quadrature formula with nodes $c_{i}(i=1, \ldots, s)$ and weights $b_{i}$ of order $p$. Let $\widehat{A}(\tau)$ be the interpolation polynomial to $A(\tau)$ in the points $c_{i} h$, and denote by $\Omega_{0}$ the expression obtained by replacing $A(\tau)$ by $\widehat{A}(\tau)$ in the expression for $\widetilde{\Omega}_{0}=\widetilde{\Omega}(h)$. Similarly, let $\widetilde{\Omega}_{n}$ and $\Omega_{n}$ denote the corresponding expressions for the $n$th step, with $A\left(t_{n}+\tau\right)$ instead of $A(\tau)$.

LEMMA 7.3. The quadrature error in the $n$th step satisfies

$$
\left\|\left(\widetilde{\Omega}_{n}-\Omega_{n}\right) v\right\| \leq C h^{p+1}\left\|D^{p-2} v\right\|
$$

The constant $C$ depends only on $M_{m}$ for $m \leq p$ and $K_{1}$.

Proof. The proof follows the lines of the proof of Lemma 6.4. The generalization concerns the appearance of $m$-fold integrals of $m$-fold iterated commutators, for $m \leq p-2$, instead of the simple commutators studied in the proof of Lemma 6.4. These terms are treated by the same techniques, they just involve more formidable expressions. By the commutator bound (3.6), this leads to an estimate involving $\left\|D^{p-2} v\right\|$ in the general situation. $\square$

As in Lemma 6.5, this implies

$$
\left\|\exp \left(\widetilde{\Omega}_{n}\right) v-\exp \left(\Omega_{n}\right) v\right\| \leq C h^{p+1}\left\|D^{p-2} v\right\| .
$$

Inserting the estimates (7.4) and (7.6) in the framework of Section 4 finally yields the error bound of Theorem 3.2. 
8. Numerical experiments. To illustrate the theoretical results presented in this paper, we consider the Schrödinger equation

$$
i \frac{\partial \psi}{\partial t}=-\frac{1}{2} \Delta \psi+b(x, t) \psi, \quad x=\left(x_{1}, \ldots, x_{d}\right) \in \mathbb{R}^{d}, t>0
$$

with a smooth $\left(C^{\infty}\right)$ potential $b(x, t)$ that is $2 \pi$-periodic in every coordinate direction $x_{j}$. We impose periodic initial conditions $\psi(x, 0)=\psi_{0}(x)$. For ease of notation only, the following discussion is for the one-dimensional case $d=1$.

A standard space discretization is given by the pseudo-spectral method. Here, a trigonometric polynomial

$$
\psi^{N}(x, t)=\sum_{k=-N / 2}^{N / 2-1} c_{k}^{N}(t) e^{i k x}
$$

is determined such that the equations

$$
\begin{aligned}
i \dot{\psi}^{N}\left(x_{\ell}, t\right) & =-\frac{1}{2} \Delta \psi^{N}\left(x_{\ell}, t\right)+b\left(x_{\ell}, t\right) \psi^{N}\left(x_{\ell}, t\right) \\
\psi^{N}\left(x_{\ell}, 0\right) & =\psi_{0}\left(x_{\ell}\right)
\end{aligned}
$$

are satisfied at the mesh-points $x_{\ell}=2 \pi \ell / N$ with $\ell=-N / 2, \ldots, N / 2-1$. Setting $c^{N}(t)=\left(c_{k}^{N}(t)\right)$ the vector of Fourier coefficients, this amounts to solving

$$
i \dot{c}^{N}=-\frac{1}{2} \widehat{\Delta}^{N} c^{N}+B^{N}(t) c^{N},
$$

where, in the case of one space dimension,

$$
\widehat{\Delta}^{N}=\left(\widehat{D}^{N}\right)^{2} \quad \text { with } \quad \widehat{D}^{N}=\operatorname{diag}(i k) \quad(k=-N / 2, \ldots, N / 2-1),
$$

and, with $F_{N}$ denoting the discrete Fourier transform of length $N$,

$$
B^{N}(t)=F_{N} \operatorname{diag}\left(b\left(x_{\ell}, t\right)\right) F_{N}^{-1} .
$$

We consider a one-dimensional example with data from [11], slightly modified to make the potential periodic with respect to the space interval $x \in[-\ell, \ell]$ for $\ell=10$ :

$$
b(x, t)=\frac{1}{2} \frac{\pi^{2}}{\ell^{2}}\left(1-\cos \frac{\pi x}{\ell}\right)+\sin ^{2}(t) \frac{\pi}{\ell} \sin \frac{\pi x}{\ell} .
$$

In the left picture of Figure 8.1 we give precision-step size diagrams at $t=1$ for four different initial values, where we used $N=128$ Fourier modes for the spatial discretization. As a smooth initial value, we used the eigenstate of the unforced harmoic oscillator to the lowest energy level, $\Psi(x, 0)=e^{-x^{2} / 2}$. The convergence curves of the exponential midpoint and the fourth-order Gauss method corresponding to the smooth initial data are the solid lines marked with circles. For the other three curves, initial data of finite energy is chosen as $c^{N}(0)=\left(I-i\left(\widehat{D}^{N}\right)^{j}\right)^{-1} v / \rho, j=1,2,3$, where $v$ is a vector of normally distributed random numbers, and $\rho$ is chosen such that $\left\|c^{N}(0)\right\|=1$. For $j=1$, the results are plotted in the dashdotted curve marked with $\times$ symbols, for $j=2$, we have the dashed curved marked with + symbols, and for $j=3$, the curve is dotted marked with diamonds.

For the right picture of Figure 8.1, we took the smooth initial state $\Psi(x, 0)=$ $e^{-x^{2} / 2}$ for all curves but varied the number of Fourier modes from $N=32$ to $N=2048$ 

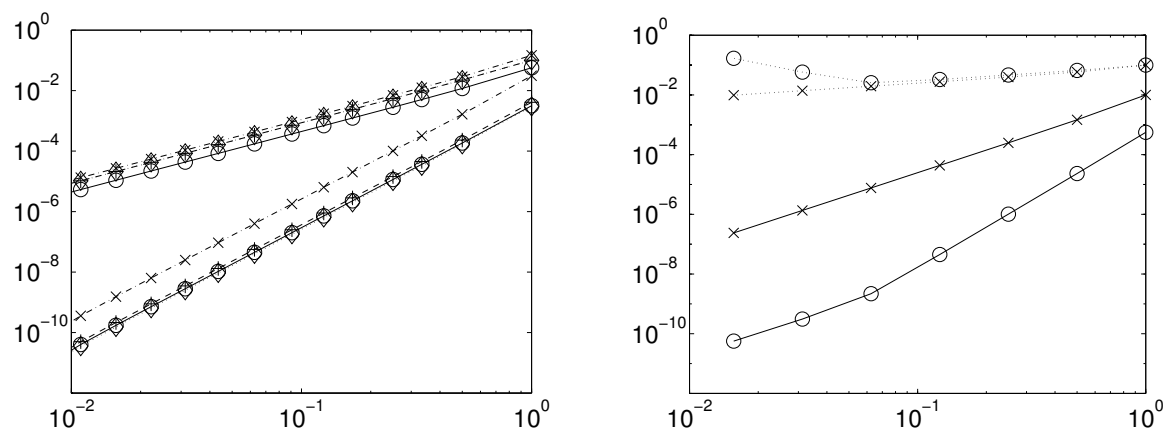

Fig. 8.1. Error versus step sizes for the laser example: smooth and nonsmooth initial data on the left, $h\|D\|=$ const on the right.

and the time steps such that $N h=32$. This corresponds to the situation that $\|h D\| \approx 3.5$, where $D=\left(-\frac{1}{2} \widehat{\Delta}^{N}+I\right)^{1 / 2}$. The solid line marked with the $\times$ symbol indicates the error of the midpoint rule, and the solid line marked with circles is the error for the fourth order Gauss method. The dotted lines in the top of the picture represent the errors of the exponential midpoint and the Gauss method divided by $h^{2}$ and $h^{4}$, respectively, up to a constant factor.

9. Appendix. Commutator bounds for a spectral discretization. We consider the pseudo-spectral space discretization (8.2) of the Schrödinger equation (8.1). Equation (8.2) is of the type studied in this paper, with $U=-\frac{1}{2}\left(\widehat{D}^{N}\right)^{2}+I$ and $V(t)=B^{N}(t)-I$. The matrix $B^{N}(t)$ is circulant, with $(k, l)$ entry equal to

$$
\widehat{b}_{k-l}^{N}(t)=\sum_{q=-\infty}^{\infty} \widehat{b}_{k-l+q N}(t)
$$

by the aliasing formula, where $\widehat{b}_{j}(t)$ is the $j$ th Fourier coefficient of the $2 \pi$-periodic (in $x$ ) function $b(x, t)$. If (and only if) $b(x, t)$ is a $C^{\infty}$ function of $x$, the Fourier coefficients $\widehat{b}_{j}(t)$ decay faster than any negative power of $|j|$. It then follows that the entries of the matrix $B^{N}(t)=\left(b_{k, l}^{N}\right)$ are bounded by

$$
\left|b_{k l}^{N}\right| \leq \begin{cases}\gamma_{m}(|k-l|+1)^{-m}, & |k-l| \leq N / 2 \\ \gamma_{m}(N-|k-l|)^{-m}, & |k-l|>N / 2\end{cases}
$$

for $k, l=-N / 2, \ldots, N / 2-1$ with $\gamma_{m}(m=1,2,3, \ldots)$ independent of $N$.

The commutator bound (3.6) is obtained as a direct consequence of the three lemmas below, for which we need to give a further definition. We say that a sequence of matrices $\mathcal{B}=\left(B^{N}\right)$, with $B^{N}$ of dimension $N \times N$, belongs to the class $\Gamma^{\infty}$, if the entries satisfy estimates (9.1) with all $\gamma_{m}$ independent of $N$. We denote by $\gamma(\mathcal{B})=\left(\gamma_{1}, \gamma_{2}, \gamma_{3}, \ldots\right)$ the sequence of smallest possible such numbers.

Lemma 9.1. If $\mathcal{A}=\left(A^{N}\right)$ and $\mathcal{B}=\left(B^{N}\right)$ are in $\Gamma^{\infty}$, then also $\mathcal{A B}=\left(A^{N} B^{N}\right)$ is in $\Gamma^{\infty}$, and $\gamma(\mathcal{A B})$ is bounded in terms of $\gamma(\mathcal{A})$ and $\gamma(\mathcal{B})$.

The proof is by direct estimation and is not given here. The following result is shown in the proof of Lemma 3.1 in [8]. 
Lemma 9.2. If $\mathcal{B}=\left(B^{N}\right)$ is in $\Gamma^{\infty}$, then $\left[\widehat{\mathcal{D}}^{2}, \mathcal{B}\right]=\left(\left[\left(\widehat{D}^{N}\right)^{2}, B^{N}\right]\right)$ is of the form

$$
\left[\widehat{\mathcal{D}}^{2}, \mathcal{B}\right]=\mathcal{M}_{0}+\mathcal{M}_{1} \widehat{\mathcal{D}}
$$

where $\mathcal{M}_{0}$ and $\mathcal{M}_{1}$ are in $\Gamma^{\infty}$, with $\gamma\left(\mathcal{M}_{0}\right)$ and $\gamma\left(\mathcal{M}_{1}\right)$ bounded in terms of $\gamma(\mathcal{B})$.

The next lemma is proved in the same way.

Lemma 9.3. If $\mathcal{B}=\left(B^{N}\right)$ is in $\Gamma^{\infty}$, then $\widehat{\mathcal{D}} \mathcal{B}=\left(\widehat{D}^{N} B^{N}\right)$ is of the form

$$
\widehat{\mathcal{D}} \mathcal{B}=\mathcal{K}_{0}+\mathcal{K}_{1} \widehat{\mathcal{D}}
$$

where $\mathcal{K}_{0}$ and $\mathcal{K}_{1}$ are in $\Gamma^{\infty}$, with $\gamma\left(\mathcal{K}_{0}\right)$ and $\gamma\left(\mathcal{K}_{1}\right)$ bounded in terms of $\gamma(\mathcal{B})$.

Repeated application of these lemmas shows that

$$
\left[-\left(\widehat{D}^{N}\right)^{2}+B^{N}\left(\tau_{k}\right),\left[\ldots,\left[-\left(\widehat{D}^{N}\right)^{2}+B^{N}\left(\tau_{1}\right), \frac{d^{m}}{d t^{m}} B^{N}\left(\tau_{0}\right)\right]\right] \ldots\right]=\sum_{j=0}^{k} M_{j}^{N}\left(\widehat{D}^{N}\right)^{j}
$$

with matrices $M_{j}^{N}$ bounded independently of $N$ and $\tau_{0}, \ldots, \tau_{k}$. This gives the desired commutator bound (3.6).

Acknowledgment. We thank Katina Lorenz for stimulating discussions on commutator bounds.

\section{REFERENCES}

[1] S. Blanes, F. Casas, J.A. Oteo, And J. Ros, Magnus and Fer expansions for matrix differential equations: the convergence problem, J. Phys. A: Math. Gen., 22 (1998), pp. 259-268.

[2] S. Blanes, F. Casas, AND J. Ros, Improved high order integrators based on the Magnus expansion, BIT, 40 (2000), pp. 434-450.

[3] S. Blanes And P.C. Moan, Splitting methods for the time-dependent Schrödinger equation, Phys. Lett., A 265 (2000), pp. 35-42.

[4] D. Henry, Geometric Theory of Semilinear Parabolic Equations, Lecture Notes in Mathematics, vol. 840, Springer, Berlin, Heidelberg, 1981.

[5] M. Hochbruck AND C. LuBich, Exponential integrators for quantum-classical molecular dynamics, BIT, 39 (1999), pp. 620-634.

[6] A. Iserles, H.Z. Munthe-KaAs, S.P. Nørsett, and A. Zanna, Lie group methods, Acta Numerica, 9 (2000), pp. 215-365.

[7] A. Iserles AND S. P. NøRSett, On the solution of linear differential equations in Lie groups, Philos. Trans. Royal. Soc. A, 357 (1999), pp. 983-1019.

[8] T. JAhnke AND C. LUBICH, Error bounds for exponential operator splittings, BIT, 40 (2000), pp. $735-744$

[9] W. Magnus, On the exponential solution of differential equations for a linear operator, Comm. Pure Appl. Math., 7 (1954), pp. 649-673.

[10] P.C. MoAn, On backward error analysis and Nekhoroshev stability in the numerical analysis of conservative systems of ODEs, PhD Thesis, Univ. of Cambridge, 2002.

[11] U. Peskin, R. Kosloff, And N. Moiseyev, The solution of the time dependent Schrödinger equation by the $\left(t, t^{\prime}\right)$ method: The use of global polynomial propagators for time dependent Hamiltonians, J. Chem. Phys., 100 (1994), pp. 8849-8855.

[12] I.V. PuZynin, A.V. Selin, S.I. Vinitsky, Magnus-factorized method for numerical solving the time-dependent Schrödinger equation, Comp. Phys. Comm., 126 (2000), pp. 158-161. 